\title{
Summarized distributions of mass: a statistical approach to consumers' consumption spaces
}

\author{
Pierpaolo Angelini ${ }^{\mathrm{a}}$ and Fabrizio Maturo ${ }^{\mathrm{b}, *}$ \\ ${ }^{a}$ Department of Statistical Sciences, Sapienza University of Rome, Italy \\ ${ }^{\mathrm{b}}$ Department of Mathematics and Physics, University of Campania "Luigi Vanvitelli", Caserta, Italy
}

\begin{abstract}
This paper focuses on logical aspects of choices being made by the consumer under conditions of uncertainty or certainty. Such logical aspects are found out to be the same. Choices being made by the consumer that should maximize her subjective utility are decisions studied by revealed preference theory. A finite number of possible alternatives is considered. They are mutually exclusive propositions identifying all quantitative states of nature of a consumption plan. Each proposition of it is expressed by a real number. This research work distinguishes it from its temporary truth value depending on the state of information and knowledge of the consumer. Since each point of the consumption space of the consumer belongs to a two-dimensional convex set, this article focuses on conjoint distributions of mass. Indeed, the consumption space of the consumer is generated by all coherent summaries of a conjoint distribution of mass. Each point of her consumption space is connected with a weighted average of states of nature of two consumption plans jointly studied. They give rise to a conjoint distribution of mass. The consumer chooses a point of a two-dimensional convex set representing that bundle of goods actually demanded by her inside of her consumption space. This paper innovatively shows that it is nothing but a bilinear and disaggregate measure. It is decomposed into two real numbers, where each real number is a linear measure. In this paper, different measures are obtained. They can be disaggregate or aggregate measures, where the latter are independent of the notion of ordered pair of consumption plans.
\end{abstract}

Keywords: 2-parallelepiped, $\alpha$-product, temporary truth value, antisymmetric tensor, non-linear metric, linear metric

\section{Introduction}

Choices being made by the consumer are directly observable. Revealed preference theory studies them as decisions that should maximize her subjective utility (see also [31], where a comprehensive approach to revealed preference theory is considered). The correct criterion of decision-making is based on logical aspects characterizing both choices being made by

\footnotetext{
${ }^{*}$ Corresponding author. Fabrizio Maturo, Department of Mathematics and Physics, University of Campania "Luigi Vanvitelli", Caserta, Italy. E-mail: fabrizio.maturo@unicampania.it.
}

the consumer under uncertainty and the ones being made by her under certainty. Such logical aspects are found out to be the same. Since the consumer actually finds herself in a state of uncertainty in almost all circumstances, the usual modes of deciding pragmatically depend on the factor uncertainty as the peerless and determinative element. We consequently realize that choices being made by the consumer under certainty are a simplification (see also [37], where the consumption theory is dealt with in terms of revealed preference). They are a particular case (see also [3], where choices under conditions of uncertainty are studied). This research work focuses on the general 
one without changing anything. It innovatively identifies a more general approach.

In this paper, choices of bundles of goods being made by the consumer are dealt with. An infinite number of alternatives cannot realistically be considered. There are not repeatable alternatives into the formulation of any choice problem concerning bundles of goods and into the arguments required for its solution. Thus, the consumer is naturally faced with a finite number of possible alternatives, where each of them is not itself subdivisible for the purposes of the problem under consideration. The linearity of their arithmetic interpretation plays a fundamental role. For this reason, all quantitative states of nature of a consumption plan are studied inside of a linear space over $\mathbb{R}$ furnished with a measure. Each of them is a possible proposition expressed by a real number. If we consider $m$ mutually exclusive states of nature then they identify an $m$-dimensional consumption vector expressed by $\mathbf{y}=\left(y^{1}, y^{2}, \ldots, y^{m}\right)$. It belongs to an $m$-dimensional linear space over $\mathbb{R}$ denoted by $E^{m}$. With regard to an orthonormal basis of $E^{m}$, we have just written the contravariant components of $\mathbf{y} \in E^{m}$ as an ordered $m$-tuple of real numbers. Each proposition is associated with a mass identifying its temporary truth value lying between 0 and 1 , end points included. The temporary truth value of a possible proposition is of a relative and subjective nature. Indeed, it depends on the state of information and knowledge of the consumer as well as her attitude towards uncertainty (see also [15], where essential psychological aspects connected with evaluations of mass are considered). Within this context, uncertainty about a state of nature of a contingent consumption plan is meant in the simple sense of ignorance by the consumer (see also [9] with respect to possible interpretations of uncertainty). Uncertainty about a state of nature of a contingent consumption plan is numerically expressed by the value of the mass associated with it (see also [28] with respect to problems concerning choices under conditions of uncertainty).

Whenever the consumer rationally chooses her bundle of goods she actually chooses a point belonging to a convex set. Since it obeys a logical criterion, this paper studies its characteristics.

We are interested in decomposing the geometric representation of the logical situation by considering the two logical elements identifying it inside of a linear space over $\mathbb{R}$. Indeed, we distinguish between real numbers and the corresponding allocation of an evaluation of mass being made by the consumer (see also [42], where behavioral aspects connected with evaluations of mass are studied). We say that in the case of logical entities expressed by real numbers the most useful arguments are not quite effective if one thinks in terms of the set of real numbers without reference to the linear space over $\mathbb{R}$ in which this set has to be embedded (see also [13], where operational definitions of notions based on criteria permitting to measure them are dealt with). Conversely, they are absolutely effective if one considers such numbers as points belonging to the set denoted by $I(Y)$ containing different logical entities all together. Accordingly, $I(Y)$ is embedded inside of a linear space over $\mathbb{R}$ (see also [10], where current researches associated with the subjectivistic conception of probability are taken into account). Regarding an evaluation of mass, it must not be absolutely inadmissible from a logical point of view. On the other hand, given two sets of quantitative states of nature of two consumption plans separately considered, we assign the arithmetic product of the two corresponding real numbers to each state of nature of a joint consumption plan characterized by a conjoint distribution of mass.

With regard to the structure of the paper, we show, in section 2, that it is appropriate to use contravariant indices together with covariant ones. In section 3 , we define the consumer's demand functions by decomposing a bilinear measure into two linear measures. In section 4, we study two contingent consumption plans that are jointly considered. In section 5 , since we study bilinear and aggregate measures obtained by defining antisymmetric tensors of order 2 , we go away from the notion of ordered pair of contingent consumption plans. In section 6 , we show that the Bravais-Pearson correlation coefficient can be obtained starting from a bilinear and aggregate measure. In section 7, we find out that the consumer's demand functions connected with two ordinary goods having downward-sloping demand curves are similarly obtained by decomposing a bilinear measure into two linear measures. In the appendix, the two theorems stated in section 5 are lastly proved.

\section{Propositions and their masses: a particular and reasonable notation}

Within this context, a function defined on a set having only a finite number of possible values coincides with $Y$ (see also [7] with respect to problems concerning previsions of random entities). $Y$ is a random quantity whose possible monetary values are given by $I(Y)=\left\{y^{1}, y^{2}, \ldots, y^{m}\right\}$, where we have 
$y^{1}<y^{2}<\ldots<y^{m}$ without loss of generality (see also [23] with regard to the notion of partition of events). If we interpret $Y$ as a function then we have to consider particular intervals of $\mathbb{R}$ such that the pre-image of any $\left[y^{i}, y^{i}\right], i=1, \ldots, m$, is a real number coinciding with $y^{i}$ and belonging to $I(Y)$ on which $Y$ is defined. The finest set of possible propositions coincides with $I(Y)$. It identifies mutually exclusive propositions (see also [8] with regard to problems involving evaluations of probability). Its extent depends on the state of information and knowledge of the consumer. That point contained in $I(Y)$ that it will occur whenever uncertainty finally ceases expresses everything there is to be stated with respect to the problem under consideration (see also [32], where an additive measure evaluating uncertainty is dealt with). Its final truth value is objectively equal to 1 .

After establishing $m$ masses denoted by $p_{1}, p_{2}, \ldots, p_{m}$, the consumer puts them on $m$ real numbers denoted by $y^{1}, y^{2}, \ldots, y^{m}$ inside of a linear space over $\mathbb{R}$ (see also [2] with respect to distributions of mass studied inside of a linear space furnished with a measure). Each proposition under consideration can be subjected to infinite translations, so it is also possible to write

$$
\left\{y^{1}+\lambda, y^{2}+\lambda, \ldots, y^{m}+\lambda\right\}
$$

with $\lambda \in \mathbb{R}$. Nevertheless, all masses associated with them do not change. This is because expectations and sensations of the consumer related to $y^{i}+\lambda$, $i=1, \ldots, m$, do not change (see also [33], where the notion of probability is studied in connection with the one of utility). Even though her expectations and sensations affect each temporary truth value, it turns out to be $p_{1}+p_{2}+\ldots+p_{m}=1$ if and only if there is coherence (see also [38] with regard to the notion of probability).

Let $E_{i}, i=1, \ldots, m$, be the generic proposition expressing in terms of statement a state of nature of a contingent consumption plan (see also [4] with respect to the nature of logical entities connected with probabilistic evaluations). The following sequence written in the form

$$
\left(y^{1}, p_{1}\right),\left(y^{2}, p_{2}\right), \ldots,\left(y^{m}, p_{m}\right)
$$

tells us that $y^{1}$ is the monetary wealth that $Y$ provides and that can be spent by the consumer if $E_{1}$ actually occurs, $\ldots, y^{m}$ is the monetary wealth that $Y$ provides and that can be spent by the consumer if $E_{m}$ actually occurs. It is clear that the mass identifying the temporary truth value of $y^{1}$ is denoted by $p_{1}, \ldots$, the mass identifying the temporary truth value of $y^{m}$ is denoted by $p_{m}$.

We use contravariant indices together with covariant ones. This is because we distinguish the two logical elements on which decisions being made by the consumer under conditions of uncertainty are based. On the other hand, contravariant and covariant components of a same vector are always the same numbers whenever we use an orthonormal basis of the linear space over $\mathbb{R}$ under consideration (see also [34] with regard to geometric aspects of logical entities). Hence, with respect to a same geometric entity, we can use both upper indices and lower ones. This thing makes no difference.

\section{Prevision bundles and their bilinear nature}

Let ${ }_{1} Y$ and ${ }_{2} Y$ be two random quantities, where the number of the possible values for each of them is equal to $m$. Let $I\left({ }_{1} Y\right)=\left\{{ }_{(1)} y^{1}, \ldots,{ }_{(1)} y^{m}\right\}$ and $I\left({ }_{2} Y\right)=\left\{{ }_{(2)} y^{1}, \ldots,{ }_{(2)} y^{m}\right\}$ be the sets of possible values for ${ }_{1} Y$ and ${ }_{2} Y$. The two vectors identifying the possible values for ${ }_{1} Y$ and ${ }_{2} Y$ are linearly independent. Firstly, two located vectors at the origin of $E^{m}$ are completely determined by their end points. Since it is possible to call an ordered $m$-tuple of real numbers either a point of $\mathcal{E}^{m}$ or a vector of $E^{m}$, it follows that $\mathcal{E}^{m}$ and $E^{m}$ are isomorphic. Secondly, if we consider all collinear vectors with regard to the two vectors identifying the possible values for ${ }_{1} Y$ and ${ }_{2} Y$ then we obtain two straight lines. It is therefore possible to go away from $E^{m}$. We are interested in considering two mutually orthogonal straight lines coinciding with the two axes of a two-dimensional Cartesian coordinate system on which an origin, a same unit of length and an orientation are established (see also [43], where essential geometric aspects connected with structures of logical entities are dealt with). On the other hand, we have elsewhere proved that all the $m$ possible values for a random quantity identifying an $m$-dimensional vector of $E^{m}$ can be studied by considering a one-dimensional straight line on which an origin, a unit of length and an orientation are established.

Moreover, we suppose that the two contingent consumption plans under consideration are logically independent, so the Cartesian product of $I\left({ }_{1} Y\right)$ and $I\left({ }_{2} Y\right)$ denoted by $I\left({ }_{1} Y\right) \times I\left({ }_{2} Y\right)$ is considered to obtain all states of nature of a joint contingent con- 
sumption plan. Their number is overall equal to $m^{2}$ (see also [35], where finitely additive measures are studied).

\subsection{Two half-lines}

Given $Y$, its weighted average is denoted by $\mathbf{P}(Y)$. Since $\mathbf{P}$ is linear, we have to write

$$
\mathbf{P}(\lambda Y)=\lambda \mathbf{P}(Y)
$$

for every real number denoted by $\lambda$. The same is true if we consider a finite number of summands, where each summand is a random quantity.

If $\lambda \in \mathbb{R}$ then we establish that $\lambda$ lies between $\lambda^{\prime}$ and $\lambda^{\prime \prime}$. Nevertheless, if this thing holds then it is also valid with regard to $\lambda Y$ in the sense that $\lambda Y$ has to lie between $\lambda^{\prime} Y$ and $\lambda^{\prime \prime} Y$. Consider we are reasoning about $\mathbf{P}(\lambda Y)$. However, we have to note that $\lambda Y$ lies between $\lambda^{\prime} Y$ and $\lambda^{\prime \prime} Y$ when and only when $I(Y)$ contains all non-negative elements. Otherwise, all of this is not true. Accordingly, since we can always write $Y=X-Z$, where we have $X=Y$ $(Y \geq 0)$ and $Z=-Y(Y \leq 0)$, we observe that these quantities are non-negative. All their possible values are non-negative numbers. This is because we have $X=Y$, if $Y>0$ and zero otherwise, whereas we have $Z=-Y$, if $Y<0$ and zero otherwise. We therefore note that the conclusion is valid for $X$ as well as for $Z$. Accordingly, the possible values for $X$ are non-negative numbers. The same is true with respect to $Z$. It follows that the conclusion is also valid for $Y=X-Z$.

It is clear that if we consider ${ }_{1} Y$ and ${ }_{2} Y$ then it is possible to write ${ }_{1} Y={ }_{1} X-{ }_{1} Z$ and ${ }_{2} Y={ }_{2} X-{ }_{2} Z$, where the possible values for ${ }_{1} Y$ and ${ }_{2} Y$ are found on two half-lines.

\subsection{Additivity and convexity of $\mathbf{P}$}

$\mathbf{P}(Y)$ is the mathematical expectation of $Y$. We write

$$
\mathbf{P}(Y+X)=\mathbf{P}(Y)+\mathbf{P}(X)
$$

as well as

$$
y^{1} \leq \mathbf{P}(Y)=y^{1} p_{1}+y^{2} p_{2}+\ldots+y^{m} p_{m} \leq y^{m},
$$

where we have $I(Y)=\left\{y^{1}, y^{2}, \ldots, y^{m}\right\}$, with $y^{1}<$ $y^{2}<\ldots<y^{m}$, because $\mathbf{P}$ is respectively additive and convex.

Since the possible values for $Y$ are found on a half-line, all coherent values of $\mathbf{P}(Y)$ obtained by considering infinite real numbers such that we have $0 \leq p_{i} \leq 1, i=1, \ldots, m$, identify a line segment whose end points are given by $y^{1}$ and $y^{m}$. This line segment is a one-dimensional convex set. On the other hand, (1) tells us that it is possible to consider a more extended line segment. It also tells us that the number of the possible values for $Y$ is the same of the one of the possible values for $X$, so their corresponding masses are the same as well. It follows that $\mathbf{P}$ has the same properties of the ones of well-behaved preferences for which more of both goods is better and averages are weakly preferred to extremes (see also [45] with regard to the notion of utility).

$\mathbf{P}$ is a linear measure whose properties tell us that any point belonging to a one-dimensional convex set is logically admissible. In other words, any point belonging to a one-dimensional convex set is admissible in terms of coherence of $\mathbf{P}$. If the consumer chooses a point identifying what is actually demanded by her for a random good then her choice is not rational whenever such a point does not belong to a convex set. Hence, given a random quantity, this choice leads to a sure loss or win. Strictly speaking, if the consumer pays $y^{m}+a$, with $a \in \mathbb{R}^{+}$, in order to receive at most $y^{m}$ at the right time then she certainly loses $y^{m}+a-y^{m}=a$. Conversely, if she pays $y^{1}-a$ in order to receive at least $y^{1}$ at the right time then she certainly wins $y^{1}-\left(y^{1}-a\right)=a$. However, we are not interested in knowing decisions providing favorable results, but we are interested in knowing decisions providing results whose consequences are undesirable in order to avoid them.

We establish the following

Definition 3.1. $\mathbf{P}$ is a linear measure such that it is possible to replace $\left\{y^{1}, y^{2}, \ldots, y^{m}\right\}$ with $\mathbf{P}(Y)$, where the latter is obtained by using those temporary truth values associated with each proposition expressed by a real number contained in $\left\{y^{1}, y^{2}, \ldots, y^{m}\right\}$. The sum of such values has to be equal to 1 , so $\mathbf{P}(Y)$ is a coherent summary identifying that proposition expressed by a real number which is actually chosen by the consumer. Each final truth value associated with a proposition of a contingent consumption plan represented by a real number contained in $\left\{y^{1}, y^{2}, \ldots, y^{m}\right\}$ objectively coincides with 0 or 1 whenever the state of information and knowledge of the consumer changes because uncertainty ceases.

We note the following

Remark 3.1. The theory of decision-making is in general interested in knowing what the decision- 
maker actually chooses. Within this context, we are especially interested in knowing what the consumer actually demands for a good. Since her rational choice coincides with a coherent summary, such a summary identifies a proposition expressed by a real number (obtained inside of a linear space over $\mathbb{R}$ ) whose truth value is equal to 1 . This is because she is indifferent to the exchange of $Y$, with $I(Y)=\left\{y^{1}, y^{2}, \ldots, y^{m}\right\}$, for $\mathbf{P}(Y)$ with certainty.

\subsection{A two-dimensional convex set}

Given two random quantities denoted by ${ }_{1} Y$ and ${ }_{2} Y$, the object of consumer choice denoted by $\left(\mathbf{P}\left({ }_{1} Y\right), \mathbf{P}\left({ }_{2} Y\right)\right)$ is of a bilinear nature (see also [22] with regard to the connection between a bundle of goods and utility). It is called a prevision bundle. Indeed, $\mathbf{P}$ stands for prevision (mathematical expectation) of a random quantity.

The budget set of the consumer is a right triangle. It is established by the negative slope of the budget line. It is also established by the two mutually orthogonal axes of a two-dimensional Cartesian coordinate system. The point $(0,0)$ where they meet, together with all those points whose coordinates are expressed by positive numbers, belongs to it. The budget constraint of the consumer expresses what she can afford (see also [19] with regard to the notion of risk connected with what the consumer prefers).

The necessary and sufficient condition for coherence of decisions is made clear by saying that the inequality between ${ }_{1} Y$ and ${ }_{2} Y$ denoted by

$$
b_{1}\left({ }_{1} Y\right)+b_{2}\left({ }_{2} Y\right) \leq b
$$

has to be satisfied by the corresponding previsions given by $\mathbf{P}\left({ }_{1} Y\right)$ and $\mathbf{P}\left({ }_{2} Y\right)$. We consequently write

$$
b_{1} \mathbf{P}\left({ }_{1} Y\right)+b_{2} \mathbf{P}\left({ }_{2} Y\right) \leq b,
$$

where $b_{1}, b_{2}$ and $b$ are positive real numbers coinciding with prices and income respectively (see also [30] to realize how some choices being made by the investor can be optimal). The budget line is given by

$$
b_{1} \mathbf{P}\left({ }_{1} Y\right)+b_{2} \mathbf{P}\left({ }_{2} Y\right)=b,
$$

so its slope coincides with

$$
-\frac{b_{1}}{b_{2}}
$$

The budget line always passes through the point having its coordinates denoted by

$$
\left(\sup I\left({ }_{1} X\right), \sup I\left({ }_{2} X\right)\right)
$$

where we have ${ }_{1} Y={ }_{1} X-{ }_{1} Z$ and ${ }_{2} Y={ }_{2} X-{ }_{2} Z$.

We consider a conjoint distribution of mass because all its coherent summaries coincide with all points of the budget set of the consumer (see also [44], where problems involving more than one variable are considered). They coincide with all points of her consumption space.

We note the following

Remark 3.2. The consumption space is generated by all coherent summaries of a conjoint distribution of mass.

A conjoint distribution of mass is summarized by means of $\mathbf{P}\left({ }_{1} Y_{2} Y\right)$, with $\mathbf{P}\left({ }_{1} Y_{2} Y\right)$ being a bilinear and disaggregate measure. We decompose it into $\mathbf{P}\left({ }_{1} Y\right)$ and $\mathbf{P}\left({ }_{2} Y\right)$ (see also [39], where decisions are based on psychological elements).

\section{We note the following}

Remark 3.3. The choice of the consumer from her budget set coincides with the choice of a point belonging to a two-dimensional convex set. Such a point is decomposed into two points belonging to two one-dimensional convex sets. To be interested in studying rational choices being made by the consumer means to be interested in studying coherent summaries.

\subsection{Average quantities of consumption}

The average quantities of consumption connected with two random goods denoted by ${ }_{1} Y$ and ${ }_{2} Y$ that are actually demanded by the consumer are expressed by $\mathbf{P}\left({ }_{1} Y\right)=\mathbf{P}\left({ }_{1} Y\right)\left[\left(b_{1}, b_{2}, b\right)\right]$ and $\mathbf{P}\left({ }_{2} Y\right)=\mathbf{P}\left({ }_{2} Y\right)\left[\left(b_{1}, b_{2}, b\right)\right]$. It is clear that they depend on objective and subjective elements. In general, what the consumer actually chooses always depends on objective and subjective elements. In particular, the subjective elements must not logically be inadmissible, so they have to be chosen in such a way that finite additivity of masses and non-negativity of each of them hold.

In this paper, we do not consider a conjoint distribution of mass only (see also [14], where psychological elements characterizing a conjoint distribution of mass are considered). This is because we are also interested in knowing aggregate measures that are established by the consumer on the basis of her state of information and knowledge. 


\section{Geometric aspects of temporary truth values}

Let $\left({ }_{1} Y,{ }_{2} Y\right)$ be an ordered pair of random quantities. We write

$$
I\left({ }_{1} Y\right)=\left\{{ }_{(1)} y^{1}, \ldots,,_{(1)} y^{m}\right\}
$$

and

$$
I\left({ }_{2} Y\right)=\left\{{ }_{(2)} y^{1}, \ldots,{ }_{(2)} y^{m}\right\},
$$

where each set is characterized by $m$ mutually exclusive propositions (see also [6], where situations characterized by incomplete information are studied). Since each proposition of $I\left({ }_{1} Y\right)$ and $I\left({ }_{2} Y\right)$ is expressed by a real number, we assign the arithmetic product of the two corresponding real numbers to each element of the Cartesian product under consideration. It follows that if a joint random quantity denoted by ${ }_{1} Y_{2} Y$ is interpreted as a function then it is written in the following form given by

$$
{ }_{1} Y{ }_{2} Y: I\left({ }_{1} Y\right) \times I\left({ }_{2} Y\right) \rightarrow \mathbb{R},
$$

where it turns out to be ${ }_{1} Y{ }_{2} Y\left(_{(1)} y^{i},{ }_{(2)} y^{j}\right)=$ ${ }_{(1)} y^{i}{ }_{(2)} y^{j}$, with $i, j=1, \ldots, m$.

Let $\mathcal{B}_{m}^{\perp}=\left\{\mathbf{e}_{i} \mid i \in I_{m}=\{1, \ldots, m\}\right\} \quad$ be an orthonormal basis of $E^{m}$. By using the Einstein notation, if we write

$$
\text { (1) } \mathbf{y}={ }_{(1)} y^{i} \mathbf{e}_{i}
$$

and

$$
\text { (2) } \mathbf{y}={ }_{\text {(2) }} y^{i} \mathbf{e}_{i}
$$

then ${ }_{(1)} \mathbf{y} \in E^{m}$ and ${ }_{(2)} \mathbf{y} \in E^{m}$ identify two $m$ dimensional vectors whose contravariant components with respect to $\mathcal{B}_{m}^{\perp}$ coincide with all elements belonging to $I\left({ }_{1} Y\right)$ and $\left.I_{2} Y\right)$ respectively.

Since ${ }_{1} Y$ and ${ }_{2} Y$ are the two marginal random quantities of ${ }_{1} Y_{2} Y$, their temporary truth values do not change when the consumer establishes the ones of ${ }_{1} Y_{2} Y$. The temporary truth values of ${ }_{1} Y$ and ${ }_{2} Y$ are mathematically expressed by using the covariant components of two $m$-dimensional vectors, where such vectors identify all marginal masses (see also [17], where coherent evaluations of mass under conditions of uncertainty are dealt with). Conversely, the covariant components of an affine tensor of order 2 represent all the masses of the joint distribution of ${ }_{1} Y$ and ${ }_{2} Y$. They have to be established by the consumer in accordance with the temporary truth values related to each proposition contained in $I\left({ }_{1} Y\right)$ and $I\left({ }_{2} Y\right)$. Hence, the temporary truth values related to each proposition contained in $I\left({ }_{1} Y\right)$ and $I\left({ }_{2} Y\right)$ represent a limitation with regard to the ones characterizing $I\left({ }_{1} Y\right) \times I\left({ }_{2} Y\right)$. It is possible to associate in an orderly manner the covariant components of an affine tensor of order 2 with the masses of the joint distribution of ${ }_{1} Y$ and ${ }_{2} Y$. Their number is overall equal to $m^{2}$. They are associated with $m^{2}$ mutually exclusive propositions. We write

$$
p=p_{i j},
$$

with $p \in E^{m} \otimes E^{m}$. If a basis of $E^{m} \otimes E^{m}$ is denoted by $\mathcal{B}_{m^{2}}=\left\{\mathbf{e}_{i} \otimes \mathbf{e}_{j} \mid(i, j) \in I_{m} \times I_{m}\right\}$ then it is possible to write

$$
p_{11} \mathbf{e}_{1} \otimes \mathbf{e}_{1}+p_{12} \mathbf{e}_{1} \otimes \mathbf{e}_{2}+\ldots+p_{m m} \mathbf{e}_{m} \otimes \mathbf{e}_{m} .
$$

We note that it turns out to be

$$
\sum_{i=1}^{m} \sum_{j=1}^{m} p_{i j}=1
$$

if and only if all evaluations being made by the consumer are coherent (see also [16] to realize that conditions of coherence are of an objective nature).

We establish the following

Definition 4.1. A bilinear and disaggregate measure denoted by $\mathbf{P}\left({ }_{1} Y_{2} Y\right)$ is obtained by considering the notion of $\alpha$-product between ${ }_{(1)} \mathbf{y}$ and ${ }_{(2)} \mathbf{y}$. It is a weighted average of states of nature of two contingent consumption plans jointly considered. It is a scalar product obtained by using all the masses of the joint distribution of ${ }_{1} Y$ and ${ }_{2} Y$ together with the contravariant components of ${ }_{(1)} \mathbf{y}$ and ${ }_{(2)} \mathbf{y}$. We write

$$
\left\langle_{(1)} \mathbf{y},{ }_{(2)} \mathbf{y}\right\rangle_{\alpha}={ }_{(1)} y^{i}{ }_{(2)} y^{j} p_{i j}=\mathbf{P}\left({ }_{1} Y_{2} Y\right) \text {. }
$$

An ordered pair of random quantities denoted by $\left({ }_{1} Y,{ }_{2} Y\right)$ is represented by an ordered triple of geometric entities denoted by

$$
\left({ }_{(1)} \mathbf{y},{ }_{(2)} \mathbf{y}, p_{i j}\right),
$$

where we have $(i, j) \in I_{m} \times I_{m}$.

\subsection{Changes of origin}

Now, we go away from the budget set of the consumer. Hence, we go away from a two-dimensional Cartesian coordinate system. Let ${ }_{Y} t$ be a random quantity whose possible values represent all deviations from $\mathbf{P}(Y)$ (see also [36], where generalized 
deviations are considered). We write

$$
\overline{\mathbf{y}}=\left(\begin{array}{c}
\bar{y}^{1}=\mathbf{P}(Y) \\
\bar{y}^{2}=\mathbf{P}(Y) \\
\vdots \\
\bar{y}^{m}=\mathbf{P}(Y)
\end{array}\right) .
$$

The contravariant components of $\overline{\mathbf{y}} \in E^{m}$ are all equal. We note that ${ }_{Y} t$ is identified with an $m$ dimensional vector of $E^{m}$ given by

$$
\mathbf{y}=\mathbf{y}-\overline{\mathbf{y}} \text {. }
$$

Its contravariant components are expressed by

$$
\mathbf{y} t^{i}=y^{i}-\bar{y}^{i}
$$

where we have $i=1, \ldots, m$.

Given ${ }_{1} Y$ and ${ }_{2} Y$, they can therefore be represented by two vectors of $E^{m}$ denoted by ${ }_{(1)} y \mathbf{t}$ and ${ }_{(2)} \mathrm{y}$ t. We write

$$
{ }_{(1)} \mathbf{y} \mathbf{t}={ }_{(1)} \mathbf{t}
$$

and

$$
{ }_{(2)} \mathbf{y} \mathbf{t}={ }_{(2)} \mathbf{t} .
$$

Given a linear combination of ${ }_{(1)} \mathbf{t}$ and ${ }_{(2)} \mathbf{t}$ expressed in the following form

$$
\mathbf{x}=\mu_{1_{(1)}} \mathbf{t}+\mu_{2(2)} \mathbf{t},
$$

where we have $\mu_{1}, \mu_{2} \in \mathbb{R}$, it is possible to write

$$
\left.\|\mathbf{x}\|_{\alpha}^{2}=\left(\mu_{1}\right)^{2}\left\|_{(1)} \mathbf{t}\right\|_{\alpha}^{2}+2 \mu_{1} \mu_{2} \chi_{(1)} \mathbf{t},{ }_{(2)} \mathbf{t}\right\rangle_{\alpha}
$$

with

$$
+\left(\mu_{2}\right)^{2}\left\|_{(2)} \mathbf{t}\right\|_{\alpha}^{2}
$$

$$
\begin{aligned}
& \left\langle_{(1)} \mathbf{t},{ }_{(1)} \mathbf{t}\right\rangle_{\alpha}=\left\|_{(1)} \mathbf{t}\right\|_{\alpha}^{2}=\operatorname{Var}\left({ }_{1} Y\right), \\
& \left.\left\langle_{(2)} \mathbf{t},{ }_{(2)} \mathbf{t}\right\rangle_{\alpha}=\left\|_{(2)} \mathbf{t}\right\|_{\alpha}^{2}=\operatorname{Var}{ }_{2} Y\right)
\end{aligned}
$$

as well as

$$
\left\langle{ }_{(1)} \mathbf{t},{ }_{(2)} \mathbf{t}\right\rangle_{\alpha}=\operatorname{Cov}\left({ }_{1} Y,{ }_{2} Y\right) .
$$

We establish the following

Definition 4.2. We call a linear metric the expression given by $\|\mathrm{x}\|_{\alpha}^{2}$. Only a joint distribution of ${ }_{1} Y$ and ${ }_{2} Y$ depending on the notion of ordered pair of contingent consumption plans is taken into account whenever we consider a linear metric.

\section{Bilinear and aggregate measures}

We note the following

Remark 5.1. If the possible values for ${ }_{1} Y$ and ${ }_{2} Y \log$ ically coincide with the contravariant components of two $m$-dimensional and linearly independent vectors of $E^{m}$ then the possible values for ${ }_{1} Y_{2} Y$ coincide with the contravariant components of an affine tensor of order 2 belonging to $E^{m} \otimes E^{m}$.

Remark 5.2. Let ${ }_{1} Y$ and ${ }_{2} Y$ be two random quantities. If we jointly consider two random quantities then an ordered pair of them is denoted by $\left({ }_{1} Y,{ }_{2} Y\right)$. If we want to study two random quantities in a joint way, but we want to go away from the notion of ordered pair of random quantities then we have to consider four ordered pairs of random quantities denoted by $\left({ }_{1} Y,{ }_{1} Y\right),\left({ }_{1} Y,{ }_{2} Y\right),\left({ }_{2} Y,{ }_{1} Y\right)$ and $\left({ }_{2} Y,{ }_{2} Y\right)$. It follows that an antisymmetric tensor of order 2 has to be defined. It is based on the notion of affine tensor of order 2. It is possible to obtain bilinear and aggregate measures whenever we consider two random quantities regardless of the notion of ordered pair.

We have to define a double random quantity. It is denoted by

$$
Y_{12}=\left\{{ }_{1} Y,{ }_{2} Y\right\}
$$

where its possible values coincide with the contravariant components of an antisymmetric tensor of order 2 (see proof of Theorem 5.1 later). It is clear that the components of $Y_{12}$ are $_{1} Y$ and ${ }_{2} Y$ (see also [29], where the ratio of two measured quantities is dealt with). After choosing $\mathrm{m}^{2}$ masses connected with ${ }_{1} Y_{2} Y$, it is necessary to consider four joint distributions characterizing ${ }_{1} Y{ }_{1} Y,{ }_{1} Y{ }_{2} Y,{ }_{2} Y{ }_{1} Y$ and ${ }_{2} Y{ }_{2} Y$, with

$$
\begin{aligned}
& { }_{1} Y{ }_{1} Y: I\left({ }_{1} Y\right) \times I\left({ }_{1} Y\right) \rightarrow \mathbb{R}, \\
& { }_{2} Y{ }_{2} Y: I\left({ }_{2} Y\right) \times I\left({ }_{2} Y\right) \rightarrow \mathbb{R}
\end{aligned}
$$

and

$$
{ }_{2} Y_{1} Y: I\left({ }_{2} Y\right) \times I\left({ }_{1} Y\right) \rightarrow \mathbb{R},
$$

in order to define $Y_{12}$ in such a way that the notion of ordered pair of random quantities is put away.

It is evident that the possible values for the marginal components of $Y_{12}$ are always considered together with their marginal masses. It follows that each distribution of mass of a marginal random quantity is viewed as a peculiar joint distribution within this context. Since we can think of putting all conjoint masses 
into a two-way table having $m$ rows and $m$ columns, all off-diagonal conjoint masses of it coincide with 0 , whereas all conjoint masses on the main diagonal of it coincide with the marginal masses of a marginal random quantity.

We prove (in the appendix) the following

Theorem 5.1. The mathematical expectation of $Y_{12}=\left\{{ }_{1} Y,{ }_{2} Y\right\}$ is a bilinear and aggregate measure coinciding with the determinant of a square matrix of order 2. Each element of such a determinant is a bilinear measure. Each element of this determinant is a real number coinciding with the mathematical expectation of ${ }_{i} Y_{j} Y$, where we have $i, j=1,2$.

Given ${ }_{1} Y$ and ${ }_{2} Y$ and their coherent previsions denoted by $\mathbf{P}\left({ }_{1} Y\right)$ and $\mathbf{P}\left({ }_{2} Y\right)$, we also consider all deviations from $\mathbf{P}\left({ }_{1} Y\right)$ and $\mathbf{P}\left({ }_{2} Y\right)$ of the possible values for ${ }_{1} Y$ and ${ }_{2} Y$.

We consequently prove (in the appendix) the following

Theorem 5.2. The variance of $Y_{12}=\left\{{ }_{1} Y,{ }_{2} Y\right\}$ coincides with the determinant of a square matrix of order 2. Each element of such a determinant is a bilinear measure. Each element of this determinant is a real number coinciding with the variance of ${ }_{1} Y$ and ${ }_{2} Y$ and with their covariance.

We observe that $\mathbf{P}\left(Y_{12}\right)$ is a coherent measure in the same way as $\mathbf{P}\left({ }_{1} Y\right), \mathbf{P}\left({ }_{2} Y\right)$ as well as $\mathbf{P}\left({ }_{1} Y_{2} Y\right)=$ $\mathbf{P}\left({ }_{2} Y_{1} Y\right)$ (see also [18] to know the real nature of such measures).

The origin of the variability of $Y_{12}$ is not random, but it depends on the state of information and knowledge of the consumer (see also [27] to realize how to measure uncertainty). All deviations from $\mathbf{P}\left({ }_{1} Y\right)$ and $\mathbf{P}\left({ }_{2} Y\right)$ of the possible values for ${ }_{1} Y$ and ${ }_{2} Y$ depend on her state of information and knowledge. This implies that the model with regard to which it is possible to compare a concrete distribution of mass is not a theoretical distribution characterized by one or more than one parameter, but it is itself a concrete distribution (see also [24], where a binomial distribution is considered).

\section{A line of argument about relevance of bilinear and aggregate measures}

We go away from the budget set of the consumer. It is known that it is possible to study consumption data outside of the budget set of the consumer. For instance, it is possible to check whether her choices maximize her utility, or do not maximize. However, we always study them inside of a linear space over $\mathbb{R}$. It has a different dimension with regard to the one of her consumption space. Everything can therefore be studied from a geometric point of view provided one takes a sufficient number of dimensions.

If we deal with two random quantities denoted by ${ }_{1} Y$ and ${ }_{2} Y$ then the Bravais-Pearson correlation coefficient is always referred to a double random quantity denoted by $Y_{12}$ (see also [21], where uncertainty is considered). Such a coefficient is a metric measure of linear correlation between two sets of possible propositions, so we consider all deviations from $\mathbf{P}\left({ }_{1} Y\right)$ and $\mathbf{P}\left({ }_{2} Y\right)$ of the possible values for ${ }_{1} Y$ and ${ }_{2} Y$ (see also [1], where a measurable property is dealt with). Given

$$
\left\|_{12} t\right\|_{\alpha}^{2}=\left|\begin{array}{cc}
\left\|_{(1)} \mathbf{t}\right\|_{\alpha}^{2} & \left\langle_{(1)} \mathbf{t},{ }_{(2)} \mathbf{t}\right\rangle_{\alpha} \\
\left\langle_{(2)} \mathbf{t},{ }_{(1)} \mathbf{t}\right\rangle_{\alpha} & \left\|_{(2)} \mathbf{t}\right\|_{\alpha}^{2}
\end{array}\right|,
$$

with ${ }_{12} t$ which is an antisymmetric tensor of order 2 representing $Y_{12}$ whenever we consider all deviations from $\mathbf{P}\left({ }_{1} Y\right)$ and $\mathbf{P}\left({ }_{2} Y\right)$ of the possible values for ${ }_{1} Y$ and ${ }_{2} Y$, we observe that if ${ }_{(1)} \mathbf{t}$ and ${ }_{(2)} \mathbf{t}$ are $\alpha$-orthogonal vectors then we obtain

$$
\left\|_{12} \hat{t}\right\|_{\alpha}^{2}=\left|\begin{array}{cc}
\left\|_{(1)} \mathbf{t}\right\|_{\alpha}^{2} & 0 \\
0 & \left\|_{(2)} \mathbf{t}\right\|_{\alpha}^{2}
\end{array}\right|=\left\|_{(1)} \mathbf{t}\right\|_{\alpha}^{2}\left\|_{(2)} \mathbf{t}\right\|_{\alpha}^{2} .
$$

Since it turns out to be

$$
\left\langle{ }_{(1)} \mathbf{t},{ }_{(2)} \mathbf{t}\right\rangle_{\alpha}=\left\langle_{(2)} \mathbf{t},{ }_{(1)} \mathbf{t}\right\rangle_{\alpha}
$$

according to commutative property of the notion of $\alpha$-product, if we want to obtain the square of $\left\langle{ }_{(1)} \mathbf{t},{ }_{(2)} \mathbf{t}\right\rangle_{\alpha}$ by using $\left\|_{12} \hat{t}\right\|_{\alpha}^{2}$ and $\left\|_{12} t\right\|_{\alpha}^{2}$ then we have to write

$$
\left[\left\langle_{(1)} \mathbf{t},{ }_{(2)} \mathbf{t}\right\rangle_{\alpha}\right]^{2}=\left\|_{12} \hat{t}\right\|_{\alpha}^{2}-\left\|{ }_{12} t\right\|_{\alpha}^{2} .
$$

The Schwarz's $\alpha$-generalized inequality derives from

$$
\left[\left\langle_{(1)} \mathbf{t},(2), \mathbf{t}\right\rangle_{\alpha}\right]^{2} \leq\left\|_{(1)} \mathbf{t}\right\|_{\alpha}^{2}\left\|_{(2)} \mathbf{t}\right\|_{\alpha}^{2},
$$

so we write

$$
\left\|_{12} \hat{t}\right\|_{\alpha}^{2}-\left\|_{12} t\right\|_{\alpha}^{2} \leq\left\|_{12} \hat{t}\right\|_{\alpha}^{2} .
$$

All of this allows us to consider the Schwarz's $\alpha$ generalized inequality in the following form given by

$$
\left|\left(\left\|_{12} \hat{t}\right\|_{\alpha}^{2}-\left\|_{12} t\right\|_{\alpha}^{2}\right)^{1 / 2}\right| \leq\left\|_{12} \hat{t}\right\|_{\alpha} .
$$


It follows that it is possible to obtain

$$
-1 \leq \frac{\left(\left\|_{12} \hat{t}\right\|_{\alpha}^{2}-\left\|_{12} t\right\|_{\alpha}^{2}\right)^{1 / 2}}{\left\|_{12} \hat{t}\right\|_{\alpha}} \leq+1,
$$

that is to say, it is possible to write

$$
-1 \leq\left(1-\frac{\left\|_{12} t\right\|_{\alpha}^{2}}{\left\|_{12} \hat{t}\right\|_{\alpha}^{2}}\right)^{1 / 2} \leq+1 .
$$

We finally realize that the above expression within the parentheses coincides with the Bravais-Pearson correlation coefficient referred to $Y_{12}$, where $\left\|_{12} t\right\|_{\alpha}^{2}$ and $\left\|_{12} \hat{t}\right\|_{\alpha}^{2}$ are two bilinear and aggregate measures. We write it in the following form expressed by

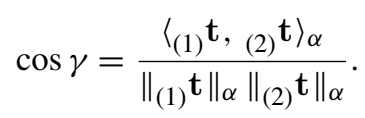

We consequently note that it always depends on the angle between $_{(1)} \mathbf{t}$ and ${ }_{(2)} \mathbf{t}$, with ${ }_{(1)} \mathbf{t},{ }_{(2)} \mathbf{t} \in E^{m}$. An appropriate numerical example verifying all of this requires some mathematical steps. For the sake of brevity, it is preferable to avoid showing them. On the other hand, we believe that it is possible to do this without leaving misunderstandings.

\section{Discussion and conclusion}

A particular case of the theory of decision-making is the consumer theory (see also [40], where an approach to demand analysis is considered). It is constructed with respect to an implicit scheme. We make it clearer by considering decisions of consumption being made by the consumer under conditions of uncertainty. We also enrich it by considering aggregate measures based on disaggregate ones. To study disaggregate and aggregate measures is equivalent to study decisions being made by the consumer under conditions of uncertainty or certainty.

With regard to decisions being made by the consumer under certainty, let $y_{1}$ and $y_{2}$ be two quantities of consumption (expressed in the same unit of measurement) of two ordinary goods of the consumer's consumption bundle denoted by $\left(y_{1}, y_{2}\right)$. It is a list of two numbers, where such numbers can also be equal. The same is true if we consider two random goods. Given two ordinary goods having downward-sloping demand curves, it is possible to say that the quantity of consumption actually demanded for each of them by the consumer under conditions of certainty is an average quantity. It is denoted by $\left(y_{1}, y_{2}\right)$. Without using the Einstein notation, we write

$$
y_{1}=y_{1}^{1} p_{1}^{1}+\ldots+y_{1}^{m} p_{1}^{m}
$$

and

$$
y_{2}=y_{2}^{1} p_{2}^{1}+\ldots+y_{2}^{m} p_{2}^{m},
$$

where $\left\{p_{1}^{i}\right\}$ and $\left\{p_{2}^{j}\right\}$ are two sets of $m$ temporary truth values viewed as masses to distribute inside of a linear space over $\mathbb{R}$ whose sum is always equal to 1 with respect to each of them. The possible quantities of consumption of good 1 are expressed by $\left\{y_{1}^{1}, \ldots, y_{1}^{m}\right\}$, whereas the possible quantities of consumption of good 2 are given by $\left\{y_{2}^{1}, \ldots, y_{2}^{m}\right\}$, where $\left\{y_{1}^{1}, \ldots, y_{1}^{m}\right\}$ and $\left\{y_{2}^{1}, \ldots, y_{2}^{m}\right\}$ are two sets containing propositions expressed by real numbers. It is clear that the consumer replaces $\left\{y_{1}^{1}, \ldots, y_{1}^{m}\right\}$ with $y_{1}$ and $\left\{y_{2}^{1}, \ldots, y_{2}^{m}\right\}$ with $y_{2}$, where $y_{1}$ and $y_{2}$ are two linear measures coinciding with two propositions expressed by two real numbers whose truth values are objectively equal to 1 . We do not go further because this thing is not currently possible unlike what happens with regard to choices under conditions of uncertainty. We are not interested in going further because we cannot observe final truth values. To say whether a proposition is true or false at the right time has no meaning within this context (see also [11] to realize how to judge consumption choices according to axioms of consumer theory). Strictly speaking, it is possible to observe $\left(y_{1}, y_{2}\right)$ at first, where $\left(y_{1}, y_{2}\right)$ is decomposed into $y_{1}$ and $y_{2}$. Afterwards, it is possible to consider the weighted average of $m^{2}$ quantities of consumption of good 1 and good 2 that are jointly considered. Given $\left\{y_{1}^{1}, \ldots, y_{1}^{m}\right\} \times\left\{y_{2}^{1}, \ldots, y_{2}^{m}\right\}$, the weighted average of $\mathrm{m}^{2}$ possible quantities of consumption of good 1 and good 2 is obtained by using $\mathrm{m}^{2}$ truth values. It is a bilinear measure that is decomposed into two linear measures (see also [20], where new developments in revealed preference theory are dealt with). We therefore consider the weighted average of $m$ possible quantities of consumption of good 1 and good 2 that are separately considered (see righthand side of (12) and (13)). All coherent weighted averages of $\mathrm{m}^{2}$ possible quantities of consumption of good 1 and good 2 that are jointly considered identify a two-dimensional convex set coinciding with a subset of a two-dimensional Cartesian coordinate system. It is the consumption space of the consumer (see also [26], where the subject of choice is studied). Moreover, they also identify two onedimensional convex sets coinciding with two line segments belonging to two mutually perpendicular 
axes of a two-dimensional Cartesian coordinate system. The budget line identifying the budget set of the consumer is given by

$$
c_{1} y_{1}+c_{2} y_{2}=c,
$$

where $c_{1}, c_{2}$ and $c$ are respectively prices and income. The consumer theory is developed with respect to this framework (see also [12], where normality of demand is studied). It is clear that one of the two goods under consideration could represent everything else the consumer wants and can afford.

In this paper, the real nature of the objects of consumer choice has been identified (see also [5], where decision-making under conditions of uncertainty is considered). We have defined two consumer's demand functions connected with two contingent consumption plans. On the other hand, such functions based on $\mathbf{P}$ have also been considered with regard to two ordinary goods actually chosen by the consumer under conditions of certainty (see also [41], where the consumer behavior is dealt with). We are interested in knowing those temporary truth values by means of which it is possible to obtain those summaries which are actually chosen by the consumer. Such values are referred to possible and mutually exclusive propositions in the case of random goods whose values can in general be of a monetary nature or not. They are conversely referred to propositions viewed as if they are mutually exclusive propositions in the case of ordinary goods. It is clear that propositions viewed as if they are mutually exclusive propositions coincide with possible propositions. The temporary truth values must not be in logical contradiction among themselves. If they are not in logical contradiction among themselves then it is possible to obtain coherent summaries. Rational choices being made by the consumer under conditions of uncertainty or certainty satisfy a logical criterion as well as maximizing her utility (see also [25] with regard to the notion of preference). These choices comply with a fundamental dichotomy between sets. Indeed, a finite number of possible alternatives gives rise to an infinite number of coherent measures summarizing them, where such measures always exist. A finite number of possible alternatives could also be embedded in a larger and more manageable space containing an infinite number of them. Nevertheless, information and knowledge of the consumer permit her to exclude many of them as impossible, so a limitation of expectations is actually carried out.
Lastly, we outline our future works. They focus on logical aspects of revealed preference theory applied to multiple random goods whose order is greater than 2. The idea of revealed preference can be measured. Prevision and utility can be measured as well. Empirical applications of revealed preference theory can be considered. How the consumer maximizes her utility associated with prevision bundles can be dealt with. On the other hand, since the scale of the budget set of the consumer is of a monetary nature, her utility associated with prevision bundles is the higher, the farther they are from the origin of her consumption space. However, it is also interesting to consider those situations where the scale of a monetary nature coincides with the one of utility.

\section{References}

[1] H. Ahmadzade, R. Gao, M.H. Dehghan and Y. Sheng, Partial entropy of uncertain random variables, Journal of Intelligent \& Fuzzy Systems 33(1) (2017), 105-112.

[2] P. Angelini and F. Maturo, Non-parametric probability distributions embedded inside of a linear space provided with a quadratic metric, Mathematics 8(11) (2020).

[3] P. Angelini and F. Maturo, The consumer's demand functions defined to study contingent consumption plans, Quality \& Quantity, (2021). https://doi.org/10.1007/s11135-02101170-2.

[4] F.J. Anscombe and R.J. Aumann, A definition of subjective probability, The Annals of Mathematical Statistics 34(1) (1963), 199-205.

[5] P. Basu and F. Echenique, On the falsifiability and learnability of decision theories, Theoretical Economics 15(4) (2020), 1279-1305.

[6] P. Battigalli and M. Siniscalchi, Rationalization and incomplete information, The B. E. Journal of Theoretical Economics 3(1) (2003).

[7] P. Berti, E. Regazzini and P. Rigo, Strong previsions of random elements, Statistical Methods and Applications (Journal of the Italian Statistical Society) 10(1) (2001), $11-28$.

[8] P. Berti and P. Rigo, On coherent conditional probabilities and disintegrations, Annals of Mathematics and Artificial Intelligence 35(1) (2002), 71-82.

[9] A. Capotorti, G. Coletti and B. Vantaggi, Standard and nonstandard representability of positive uncertainty orderings, Kybernetika 50(2) (2014), 189-215.

[10] G. Cassese, P. Rigo and B. Vantaggi, A special issue on the mathematics of subjective probability, Decisions in Economics and Finance 43(1) (2020), 1-2.

[11] C.P. Chambers, F. Echenique and E. Shmaya, General revealed preference theory, Theoretical Economics 12(2) (2017), 493-511.

[12] L. Cherchye, T. Demuynck and B. De Rock, Normality of demand in a two-goods setting, Journal of Economic Theory 173 (2018), 361-382.

[13] G. Coletti, D. Petturiti and B. Vantaggi, When upper conditional probabilities are conditional possibility measures, Fuzzy Sets and Systems 304 (2016), 45-64. 
[14] G.B. Davies and S.E. Satchell, The behavioural components of risk aversion, Journal of Mathematical Psychology 51(1) (2007), 1-13.

[15] B. de Finetti, The role of "Dutch Books" and of "proper scoring rules", The British Journal of Psychology of Sciences 32 (1981), 55-56.

[16] B. de Finetti, Probability: the different views and terminologies in a critical analysis. In Cohen, L. J., Ło's, J., H. Pfeiffer and Podewski, K.-P., editors, Logic, Methodology and Philosophy of Science VI, (1982a), pp. 391-394. North-Holland Publishing Company, Amsterdam.

[17] B. de Finetti, The proper approach to probability. In Koch, G. and Spizzichino, F., editors, Exchangeability in Probability and Statistics, (1982b). pp. 1-6. North-Holland Publishing Company, Amsterdam.

[18] B. de Finetti, Probabilism: a critical essay on the theory of probability and on the value of science, Erkenntnis 31(2-3) (1989), 169-223.

[19] S. Drapeau and M. Kupper, Risk preferences and their robust representation, Mathematics of Operations Research 38(1) (2013), 28-62.

[20] F. Echenique, New developments in revealed preference theory: decisions under risk, uncertainty, and intertemporal choice, Annual Review of Economics 12(1) (2020), 299-316.

[21] R. Gao, H. Ahmadzade, K. Rezaei, H. Rezaei and H. Naderi, Partial similarity measure of uncertain random variables and its application to portfolio selection, Journal of Intelligent \& Fuzzy Systems 39(1) (2020), 155-166.

[22] P. Ghirardato, F. Maccheroni and M. Marinacci, Certainty independence and the separation of utility and beliefs, Journal of Economic Theory 120(1) (2005), 129-136.

[23] A. Gilio and G. Sanfilippo, Conditional random quantities and compounds of conditionals, Studia Logica 102(4) (2014), 709-729.

[24] H. Haghbin, M.R. Mahmoudi and Z. Shishebor, Large sample inference on the ratio of two independent binomial proportions, Journal of Mathematical Extension 5(1) (2010), 87-95.

[25] Y. Halevy, D. Persitz and L. Zrill, Parametric recoverability of preferences, Journal of Political Economy 126(4) (2018), $1558-1593$.

[26] E.J. Johnson and J.W. Payne, Effort and accuracy in choice, Management Science 31(4) (1985), 395-414.

[27] K. Jurado, S.C. Ludvigson and S. Ng, Measuring uncertainty, American Economic Review 105(3) (2015), 1177-1216.

[28] M.J. Machina, Choice under uncertainty: problems solved and unsolved, Journal of Economic Perspectives 1(1) (1987), 121-154.
[29] M.R. Mahmoudi, J. Behboodian and M. Maleki, Large sample inference about the ratio of means in two independent populations, Journal of Statistical Theory and Applications 16(3) (2017), 366-374.

[30] H. Markowitz, The utility of wealth, Journal of Political Economy 60(2) (1952), 151-158.

[31] H. Nishimura, E.A. Ok and J.K.-H. Quah, A comprehensive approach to revealed preference theory, American Economic Review 107(4) (2017), 1239-1263.

[32] R.J. Nunke and L.J. Savage, On the set of values of a nonatomic, finitely additive, finite measure, Proceedings of the American Mathematical Society 3(2) (1952), 217-218.

[33] J. Pfanzagl, Subjective probability derived from the Morgenstern-von Neumann utility theory. In Shubik, M., editor, Essays in mathematical economics in honor of Oskar Morgenstern, (1967), pp. 237-251. Princeton University Press, Princeton.

[34] G. Pompilj, On intrinsic independence, Bulletin of the International Statistical Institute 35(2) (1957), 91-97.

[35] E. Regazzini, Finitely additive conditional probabilities, Rendiconti del Seminario Matematico e Fisico di Milano 55(1) (1985), 69-89.

[36] R.T. Rockafellar, S. Uryasev and M. Zabarankin, Generalized deviations in rịsk analysis, Finance and Stochastics $\mathbf{1 0}$ (2006), 51-74.

[37] P.A. Samuelson, Consumption theory in terms of revealed preference, Economica 15(60) (1948), 243-253.

[38] D. Schmeidler, Subjective probability and expected utility without additivity, Econometrica 57(3) (1989), 571-587.

[39] P. Slovic, B. Fischhoff and S. Lichtenstein, Behavioral decision theory, Annual Review of Psychology 28 (1977), 1-39.

[40] H.R. Varian, The nonparametric approach to demand analysis, Econometrica 50(4) (1982), 945-973.

[41] H.R. Varian, Non-parametric tests of consumer behaviour, The Review of Economic Studies 50(1) (1983), 99-110.

[42] W.K. Viscusi and W.N. Evans, Behavioral probabilities, Journal of Risk and Uncertainty 32(1) (2006), 5-15.

[43] J. von Neumann, Examples of continuous geometries, Proceedings of the National Academy of Sciences of the United States of America 22(2) (1936), 101-108.

[44] D. von Rosen, Maximum likelihood estimators in multivariate linear normal models, Journal of Multivariate Analysis 31(2) (1989), 187-200.

[45] H. Wold, G.L.S. Shackle and L.J. Savage, Ordinal preferences or cardinal utility? Econometrica 20(4) (1952), 661-664. 


\section{Appendix}

\section{A. Proof of Theorem 6.1}

Proof. Given a basis of $E^{m} \otimes E^{m}$ expressed by $\mathcal{B}_{m^{2}}=\left\{\mathbf{e}_{i} \otimes \mathbf{e}_{j} \mid(i, j) \in I_{m} \times I_{m}\right\}$, an affine tensor of order 2 can be written in the form

$$
T={ }_{(1)} \mathbf{y} \otimes{ }_{(2)} \mathbf{y}={ }_{(1)} y^{i}{ }_{(2)} y^{j} \mathbf{e}_{i} \otimes \mathbf{e}_{j}
$$

or in the form

$$
T={ }_{(2)} \mathbf{y} \otimes{ }_{(1)} \mathbf{y}={ }_{(2)} y^{j}{ }_{(1)} y^{i} \mathbf{e}_{j} \otimes \mathbf{e}_{i},
$$

where the former corresponds to $\left({ }_{1} Y,{ }_{2} Y\right)$, whereas the latter corresponds to $\left({ }_{2} Y,{ }_{1} Y\right)$. A same affine tensor of order 2 denoted by $T$ whose $m^{2}$ contravariant components are not the same has therefore been written. Since we want to go away from the notion of ordered pair of contingent consumption plans, we have to consider (15) and (16) together. Thus, we write an antisymmetric tensor of order 2 in the form

$$
T=\sum_{i<j}\left({ }_{(1)} y_{(2)}^{i} y^{j}-{ }_{(1)} y^{j}{ }_{(2)} y^{i}\right) \mathbf{e}_{i} \otimes \mathbf{e}_{j},
$$

where $i<j$ under the summation symbol means that we avoid 0 inside parentheses. It is obtained whenever we have $i=j$. We denote by ${ }_{12} y$ an antisymmetric tensor of order 2 identifying $Y_{12}$ from a logical point of view. We write

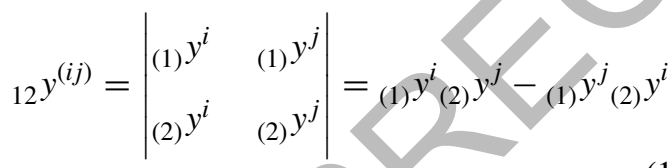

to identify the strict contravariant components of it. We have $i<j$. The number of such components is overall equal to

$$
\longrightarrow\left(\begin{array}{l}
m \\
2
\end{array}\right) \text {. }
$$

The corresponding strict covariant components of ${ }_{12} y$ are given by

$$
{ }_{12} y_{(i j)}=\left|\begin{array}{ll}
{ }_{(1)} y_{i} & \text { (1) } y_{j} \\
{ }_{(2)} y_{i} & \text { (2) } y_{j}
\end{array}\right|=\left|\begin{array}{ll}
{ }_{(1)} y^{j} p_{j i} & \text { (1) } y^{i} p_{i j} \\
{ }_{(2)} y^{j} p_{j i} & \text { (2) } y^{i} p_{i j}
\end{array}\right|,
$$

where we have $i<j$. They are characterized by vector homographies being made by using those conjoint masses belonging to a row or column of a two-way table having $m$ rows and $m$ columns. We do not compute the scalar value of (19). The number of the strict contravariant and covariant components of ${ }_{12} y$ is inessential because we always obtain the same outcome regardless of such a number. We always put together (18) and (19) in the same way. We always associate ${ }_{(1)} y^{i}$ with ${ }_{(1)} y_{i},{ }_{(1)} y^{j}$ with ${ }_{(2)} y_{j}$, (2) $y^{i}$ with ${ }_{(1)} y_{i}$ and ${ }_{(2)} y^{j}$ with ${ }_{(2)} y_{j}$. After putting together (18) and (19), we obtain different monomials for which the Einstein notation works. We consider two determinants because we are studying multilinear relationships. We obtain the mathematical expectation of $Y_{12}$ given by

$$
\begin{aligned}
\left\|_{12} y\right\|_{\alpha}^{2} & =\left|\begin{array}{cc}
\left\|_{(1)} \mathbf{y}\right\|_{\alpha}^{2} & \left\langle_{(1)} \mathbf{y},{ }_{(2)} \mathbf{y}\right\rangle_{\alpha} \\
\left\langle_{(2)} \mathbf{y},{ }_{(1)} \mathbf{y}\right\rangle_{\alpha} & \left\|_{(2)} \mathbf{y}\right\|_{\alpha}^{2}
\end{array}\right|, \\
& =\left\|_{(1)} \mathbf{y}\right\|_{\alpha}^{2}\left\|_{(2)} \mathbf{y}\right\|_{\alpha}^{2}-\left(\left\langle_{(1)} \mathbf{y},{ }_{(2)} \mathbf{y}\right\rangle_{\alpha}\right)^{2}
\end{aligned}
$$

where we evidently observe

$$
\left\langle_{(1)} \mathbf{y},{ }_{(2)} \mathbf{y}\right\rangle_{\alpha}=\left\langle_{(2)} \mathbf{y},{ }_{(1)} \mathbf{y}\right\rangle_{\alpha} .
$$

By putting together (18) and (19), we are always faced with four joint distributions characterizing ${ }_{1} Y{ }_{1} X,{ }_{1} Y{ }_{2} X,{ }_{2} Y{ }_{1} Y$ and ${ }_{2} Y{ }_{2} Y$. They are all summarized. We write

$$
\left\|_{12} y\right\|_{\alpha}^{2}=\mathbf{P}\left(Y_{12}\right),
$$

where it turns out to be

$$
\begin{aligned}
\mathbf{P}\left(Y_{12}\right) & =\left|\begin{array}{cc}
\left\|_{(1)} \mathbf{y}\right\|_{\alpha}^{2} & \left\langle_{(1)} \mathbf{y},{ }_{(2)} \mathbf{y}\right\rangle_{\alpha} \\
\left\langle_{(2)} \mathbf{y},{ }_{(1)} \mathbf{y}\right\rangle_{\alpha} & \left\|_{(2)} \mathbf{y}\right\|_{\alpha}^{2}
\end{array}\right| \\
& =\left|\begin{array}{ll}
{ }_{(1)} y^{i}{ }_{(1)} y^{i} p_{i i}^{(11)} & { }_{(1)} y^{j}{ }_{(2)} y^{i} p_{i j}^{(12)} \\
{ }_{(2)} y^{i}{ }_{(1)} y^{j} p_{j i}^{(21)} & { }_{(2)} y^{j}{ }_{(2)} y^{j} p_{j j}^{(22)}
\end{array}\right| .
\end{aligned}
$$

We note that $p^{(11)}$ is the tensor of all conjoint masses associated with $\left({ }_{1} \mathbf{y},{ }_{1} \mathbf{y}\right), p^{(12)}$ is the tensor of all conjoint masses associated with $\left({ }_{1} \mathbf{y},{ }_{2} \mathbf{y}\right)$ and so on. It is possible to observe that, in general, it turns out to be

$$
\mathbf{P}\left({ }_{1} Y_{2} Y\right) \neq \mathbf{P}\left(Y_{12}\right) .
$$

We finally write

$$
\mathbf{P}\left(Y_{12}\right)=\left|\begin{array}{ll}
\mathbf{P}\left({ }_{1} Y_{1} Y\right) & \mathbf{P}\left({ }_{1} Y_{2} Y\right) \\
\mathbf{P}\left({ }_{2} Y_{1} Y\right) & \mathbf{P}\left({ }_{2} Y_{2} Y\right)
\end{array}\right|,
$$


where the determinant of the square matrix of order 2 under consideration is a bilinear function of the columns of it.

\section{B. Proof of Theorem 6.2}

Proof. All deviations from $\mathbf{P}\left({ }_{1} Y\right)$ and $\mathbf{P}\left({ }_{2} Y\right)$ of the possible values for ${ }_{1} Y$ and ${ }_{2} Y$ are translations. It is then possible to write

$$
\begin{aligned}
\left\|_{12} t\right\|_{\alpha}^{2} & =\left|\begin{array}{cc}
\left\|_{(1)} \mathbf{t}\right\|_{\alpha}^{2} & \left\langle_{(1)} \mathbf{t},{ }_{(2)} \mathbf{t}\right\rangle_{\alpha} \\
\left\langle_{(2)} \mathbf{t},{ }_{(1)} \mathbf{t}\right\rangle_{\alpha} & \left\|_{(2)} \mathbf{t}\right\|_{\alpha}^{2}
\end{array}\right| \\
& =\left\|_{(1)} \mathbf{t}\right\|_{\alpha}^{2}\left\|_{(2)} \mathbf{t}\right\|_{\alpha}^{2}-\left(\left\langle_{(1)} \mathbf{t},{ }_{(2)} \mathbf{t}\right\rangle_{\alpha}\right)^{2} .
\end{aligned}
$$

We write

$$
\left\|_{12} t\right\|_{\alpha}^{2}=\operatorname{Var}\left(Y_{12}\right)=\sigma_{Y_{12}}^{2}
$$

We note that it turns out to be

$$
\begin{aligned}
& \left\langle{ }_{(1)} \mathbf{t},{ }_{(2)} \mathbf{t}\right\rangle_{\alpha}=\left\langle{ }_{(2)} \mathbf{t},{ }_{(1)} \mathbf{t}\right\rangle_{\alpha} \\
& =\operatorname{Cov}\left({ }_{1} Y,{ }_{2} Y\right)=\operatorname{Cov}\left({ }_{2} Y,{ }_{1} Y\right),
\end{aligned}
$$

so it is possible to write

$$
\operatorname{Var}\left(Y_{12}\right)=\left|\begin{array}{cc}
\operatorname{Var}\left({ }_{1} Y\right) & \operatorname{Cov}\left({ }_{1} Y,{ }_{2} Y\right) \\
\operatorname{Cov}\left({ }_{2} Y,{ }_{1} Y\right) & \operatorname{Var}\left({ }_{2} Y\right)
\end{array}\right| .
$$

We call a non-linear metric the expression given by (26). It is the area of a 2-parallelepiped whose edges are two single random quantities having their possible values that are subjected to two changes of origin. 